\title{
Apoptotic effects of signal transduction inhibitors on human tumor cells with different PTEN expression
}

\author{
GEORG PFEILER, FELICITAS HORN, CLAUS LATTRICH, STEFANIE KLAPPENBERGER, \\ OLAF ORTMANN and OLIVER TREECK

\begin{abstract}
Department of Obstetrics and Gynecology, University of Regensburg, Caritas Hospital St. Josef, Landshuter Strasse 65, 93053 Regensburg, Germany
\end{abstract}

Received February 12, 2007; Accepted May 16, 2007

\begin{abstract}
An important mechanism of antitumoral targeted therapies is the induction of apoptosis in tumor cells. Tamoxifen and trastuzumab (Herceptin), respectively, are able to trigger apoptosis in human breast cancer cells. But, frequently altered apoptotic signal cascades, for instance through PTEN mutations, help tumor cells to escape antitumoral therapy. We studied to what extent the apoptotic effect of signal-transduction inhibitors is dependent on PTEN expression. PTEN expression was analysed by Western blot analysis in tumor cell lines of the breast (BT-474, MCF-7, MDA-MB-231), ovary (BG-1, SK-OV-3) and endometrium (Ishikawa, HEC-1A). Apoptotic effects of tamoxifen, trastuzumab, ZD1839 (Iressa) and different mitogen-activated protein kinase (MAP) inhibitors were measured after $24 \mathrm{~h}$ of treatment. Cellular apoptosis was determined by the detection of cytoplasmic histone-DNA complexes. The tested tumor cell lines exhibited a different PTEN expression, ranging from a high expression (ovarian cancer cell line BG-1 and BT-474 breast cancer cells) to a total absence of PTEN expression (endometrial Ishikawa cells). The apoptotic effect of receptor-targeting drugs (tamoxifen, trastuzumab, ZD1839) was dependent both on receptor expression and PTEN expression. When cells were treated with MAPK inhibitors, no correlation between PTEN expression and the apoptosis rate was observed. Our data underline the importance of PTEN expression regarding the induction of apoptosis through various targeted therapies.
\end{abstract}

\section{Introduction}

Much of the current effort in anticancer drug development focuses on inhibition of different signal transduction pathways. Additionally to well-established receptor targeted drugs such as tamoxifen or trastuzumab, currently several 'small

Correspondence to: Dr O. Ortmann, Department of Obstetrics and Gynecology, University of Regensburg, Caritas Hospital St. Josef, Landshuter Strasse 65, 93053 Regensburg, Germany

E-mail: otreeck@caritasstjosef.de

Key words: PTEN, mitogen-activated protein kinase, apoptosis, cancer cells, targeted therapy molecule inhibitors' are developed, which show antitumoral action through the inhibition of cytoplasmatic kinases like mitogen-activated protein (MAP) kinases. Some of antitumoral action of targeted therapies is mediated by the induction of cellular apoptosis. Several studies have shown that both the selective estrogen modulator (SERM) tamoxifen and the humanized Her2/neu antibody trastuzumab are able to trigger cellular apoptosis in breast cancer cells $(1,2)$. However, programmed cell death in tumor cells is frequently disturbed because of mutations in the tumor suppressor gene PTEN.

PTEN is one of the most common targets for mutation in sporadic human cancers. PTEN mutations are detected in $33-83 \%$ of endometrial cancers, but also in $20-55 \%$ of endometrial hyperplasia $(3,4)$, suggesting that PTEN might be involved in the initial stages of tumor development. PTEN mutations can also be found in breast and ovarian cancer (5-30\% and 9\%, respectively) $(5,6)$. The lipid phosphatase branch of the dual phosphatase PTEN counteracts phosphatidyl-inositol-3-kinase (PI3K) by dephosphorylation of phosphoinositol 3-phosphat (PIP3) at the D3 position (7). Thereby, PTEN prevents the activation of phosphoinositidedependent kinase-1 (PDK-1) phosphorylation and the activation of AKT. The consequences of AKT inhibition includes the stimulation of apoptosis and the inhibition of cell cycle progression. The protein phosphatase branch downregulates cyclin D1, which supports the antiproliferative function of the lipid phosphatase branch, but plays a minor part of PTEN antitumoral action (8). Beyond the AKT pathway, PTEN exerts its antitumoral effects by a blockade of Shc phosphorylation resulting in the inhibition of ERK1/2 MAPK pathways leading to the inhibition of proliferation and induction of apoptosis $(9,10)$, whereas the pro-apoptotic Jnkand p38 pathways are not affected.

PTEN mutations leading to overactivity of anti-apoptotic pathways could therefore impair the apoptotic action of targeted therapies. To address this question, we investigated the apoptotic efficacy of both receptor-targeted inhibitors and small molecule inhibitors on human cancer cells with different levels of PTEN expression.

\section{Materials and methods}

Materials. 4-OH tamoxifen was obtained from Sigma-Aldrich (Munich, Germany). MEK inhibitors PD98059 and U0126 and 
Table I. Receptor expression of cell lines.

\begin{tabular}{lccc}
\hline & ER $\alpha$ & Her-2/neu & EGFR \\
\hline BT-474 & + & +++ & + \\
MCF-7 & + & + & + \\
MDA-MB-231 & - & $+/-$ & + - \\
BG-1 & + & $+/-$ & + \\
SK-OV-3 & + (mut) & +++ & +++ \\
Ishikawa & + & + & + \\
HEC-1A & $+/-$ & + & + \\
\hline
\end{tabular}

+++, overexpression; +, high expression; +/-, low expression; -, no verified expression; mut, mutated, not functional receptor.

a)
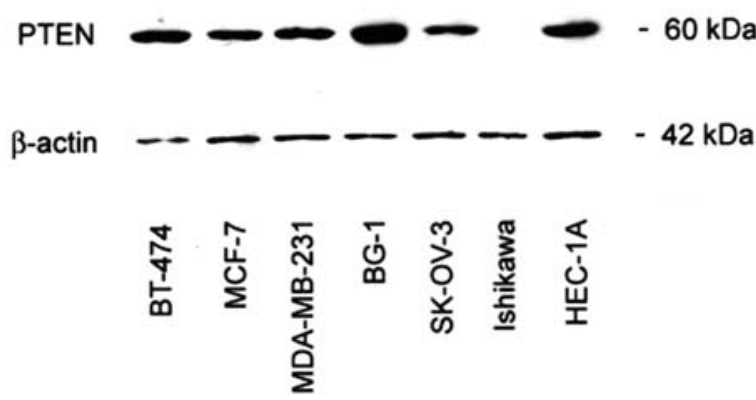

b)

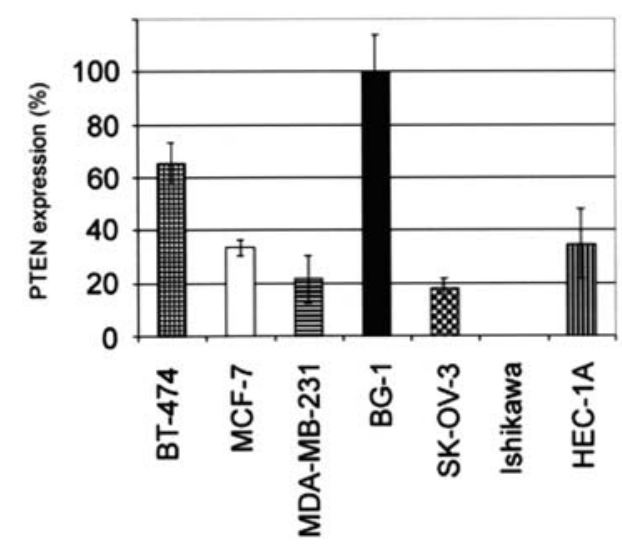

Figure 1. Western blot analysis of PTEN expression in a panel of seven cancer cell lines derived from breast, ovarian and endometrial cancer. a) Representative Western blot, $\beta$-actin expression was used as a loading control. b) Quantification of PTEN expression after the densitometric measurement of Western blot bands $(n=4)$. The relative PTEN expression is shown after normalization to the respective $B$-actin band in percent of BG-1 .

p38 MAP kinase inhibitors PD169,316 and SB202190 were obtained from Calbiochem (Darmstadt, Germany). EGFRinhibitor ZD1839 (Iressa $^{\circledR}$ ) was kindly provided by Astra Zeneca and trastuzumab (Herceptin ${ }^{\circledR}$ ) was kindly provided by Roche. Cell Death Detection ELISA Plus for apoptosis quantification was obtained from Roche (Germany). Breast cancer cell lines MCF-7, MDA-MB-231 and BT-474, ovarian cancer cell lines BG-1 and SK-OV-3 and endometrial adenocarcinoma cell lines Ishikawa and HEC-1A were obtained from ATCC (Manassas, USA). B-actin antibody (8226) and PTEN antibody (2453) were from Abcam (Cambridge, UK), ECL System from Amersham (Buckinghamshire, UK).

Cell culture and apoptosis assays. All cell lines were cultured in DMEM/F12 supplemented with $10 \% \mathrm{FCS}$ at $37^{\circ} \mathrm{C}$ in an incubator with $5 \% \mathrm{CO}_{2}$ humidified atmosphere. $5 \times 10^{3}$ cells per well of a 96-well plate were seeded and treated $24 \mathrm{~h}$ later with the following drugs: 4-OH tamoxifen $(10 \mu \mathrm{M})$, PD98059 $(10 \mu \mathrm{M})$, U0126 $(5 \mu \mathrm{M})$, PD169,136 $(10 \mu \mathrm{M}), \mathrm{SB} 202190$ $(1 \mu \mathrm{M}), \mathrm{ZD} 1839(0.1 \mu \mathrm{M})$ und Trastuzumab $(10 \mu \mathrm{g} / \mathrm{ml})$. Eighteen hours later, cellular apoptosis was determined by means of the Cell Death Detection ELISA (Roche) according to the manufacturer's instructions. This assay is based on ELISA-mediated colorimetric detection (405 nm) of cytoplasmic histone-DNA complexes. Cellular apoptosis is expressed in percent of the untreated control, statistical analysis was performed using ANOVA by means of the software Prism 2.0 (Graph pad, San Diego, USA).

Western blot analysis. Cells were lysed in RIPA (1\% Igepal CA-630, 0.5\% sodium deoxycholate, $0.1 \%$ SDS in PBS with aprotinin and sodium orthovanadate). Aliquots of $10 \mu \mathrm{g}$ protein were resolved in $10 \%$ SDS-PAGE followed by electrotransfer to a nitrocellulose membrane. Immunodetection was carried out using a PTEN- and a $\beta$-actin antibody (Abcam, Cambridge, UK) in PBS (5\% skim milk) and the ECL-system. Antibody dilutions were 1:5000 (B-actin) and 1:500 (PTEN). Band intensity was quantified using the EasyWin Software (Herolab, Wiesloch, Germany), statistical analysis was performed using ANOVA by means of the software Prism 2.0.

\section{Results}

PTEN expression in the employed cancer cell lines. To investigate the relationship between PTEN expression and apoptotic efficacy of various signal transduction inhibitors, we first analysed PTEN expression in a panel of human cancer cell lines by means of Western blot analysis. In this report, we employed cell lines of breast (BT-474, MCF-7, MDA-MB231), ovarian (BG-1, SK-OV-3) and endometrial cancer (Ishikawa, HEC-1A) also characterized by a specific expression of ER $\alpha$, EGFR and Her2/neu receptor (Table I).

PTEN expression was high in BT-474 breast cancer cells, whereas it was less pronounced in MCF-7 and MDA-MB-231 cells. The highest PTEN expression was detected in the ovarian cancer cell line BG-1, while there was only a slight expression of the tumor suppressor gene observed in SK-OV-3 cells. The endometrial cancer cell line HEC-1A exhibited a high PTEN expression, but there was no PTEN expression detected in Ishikawa cells (Fig. 1).

Apoptotic action of signal transduction inhibitors. To investigate apoptotic action of the active tamoxifen metabolite 4-OH tamoxifen, monoclonal Her2/neu antibody trastuzumab, 
EGFR inhibitor ZD1839 (Iressa) as well as of Erk-inhibitors U0126 and PD98059 and p38 kinase-inhibitors SB202190 and PD169,316, respectively, human cancer cells were treated with standard concentrations of the mentioned substances.

Treatment with $10 \mu \mathrm{M} 4-\mathrm{OH}$ tamoxifen significantly increased apoptosis in breast and ovarian cancer cells with a high expression of ER $\alpha$. In this manner, a considerable induction of apoptosis was detected in ER $\alpha$-positive BT-474 and MCF-7 cells, respectively, as well as in ER $\alpha$-positive BG-1 cells, whereas this could not be shown in ER $\alpha$-negative MDA-MB-231 and SK-OV-3 cells. In the employed endometrial adenocarcinoma cell lines, a different tamoxifen response was observed. Tamoxifen was not able to induce apoptosis in ER $\alpha$-positive but PTEN-negative Ishikawa cells, whereas the induction of apoptosis could be detected in ER $\alpha$ positive and PTEN-positive HEC-1A cells (Fig. 2).

Treatment with trastuzumab $(10 \mu \mathrm{g} / \mathrm{ml})$ considerably triggered apoptosis only in Her2/neu overexpressing BT-474 breast cancer cells also exhibiting a strong PTEN expression. No other cell line showed increased induction of apoptosis after treatment with trastuzumab. Even Her2/neu overexpressing SK-OV-3 cells, which showed a significantly reduced expression of PTEN compared to BT-474 cells, were not affected by trastuzumab. Similar results could be observed after treatment with EGFR inhibitor ZD1839. Only cells exhibiting both high PTEN and high EGFR expression showed an increased apoptosis after treatment with this drug. Increased apoptosis rates after treatment with ZD1839 could be detected in BT-474, MCF-7 and BG-1 cells with high levels of PTEN expression, whereas no such effect was observed in MDA-MB-231, SK-OV-3 and Ishikawa cells with a low or even absent PTEN expression (Fig. 2).

The p38 kinase inhibitor SB202190 was not able to induce apoptosis in any cell line tested and PD169,316 induced apoptosis only in the ovarian BG-1 cell line exhibiting high levels of PTEN expression.

Treatment with MEK Inhibitors PD98059 and U0126 led to significantly increased apoptosis rates in PTEN-positive MCF-7 breast cancer cells. For U0126, this was also observed in PTEN-positive endometrial HEC-1A cells. The apoptosis rate of the other cell lines, regardless of their PTEN expression, was not affected by treatment with one of the Erkinhibitors (Fig. 3).

\section{Discussion}

We investigated the apoptotic efficacy of various signal transduction inhibitors on human cancer cells with different PTEN expression. PTEN as a tumor suppressor inhibits not only the AKT pathway, which promotes cell growth and proliferation whereas apoptosis is inhibited, but also influences the MAPK pathway. Gu et al showed that PTEN specifically blocks the Ras/Raf/Erk pathway through Shc, whereas the Jnk and p38 MAPK signalling is not affected (10). Therefore, decreased PTEN expression would lead to overactivity of the Erk pathway. In studies on mTOR inhibitor rapamycin, it was observed that the inhibitory efficacy of mTOR was maximal when cells exhibited no PTEN expression and therefore the AKT pathway was constitutively activated (11). On the other hand, when cells showed high PTEN expression and
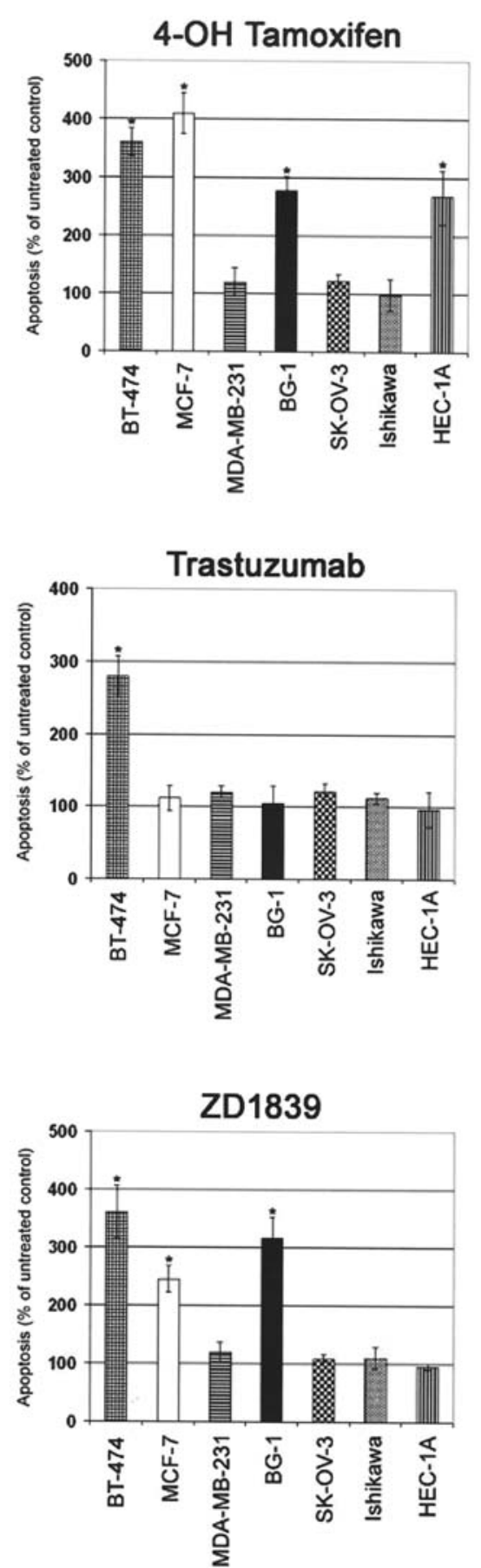

Figure 2. Apoptosis triggered by receptor-targeting antitumoral drugs. Apoptosis was assessed by the detection of cytoplasmic histone-DNA complexes and is expressed as a percent of the untreated control $(n=4)$. $* \mathrm{p}<0.01$ vs untreated control.

therefore decreased AKT signalling, rapamycin had hardly any effect on the proliferation of these cells.

We investigated the apoptotic action of MEK-inhibitors U0126 and PD98059 in relation to PTEN expression. We were not able to detect any correlation between PTEN expression and apoptosis rate after treatment with MEK inhibitors. The endometrial cancer cell line Ishikawa, in which PTEN expression was detected and therefore Erk signalling is expected to be overactivated, showed no increased apoptosis rate after treatment with U0126 and PD98059, respectively. Given that Ishikawa cells have an 

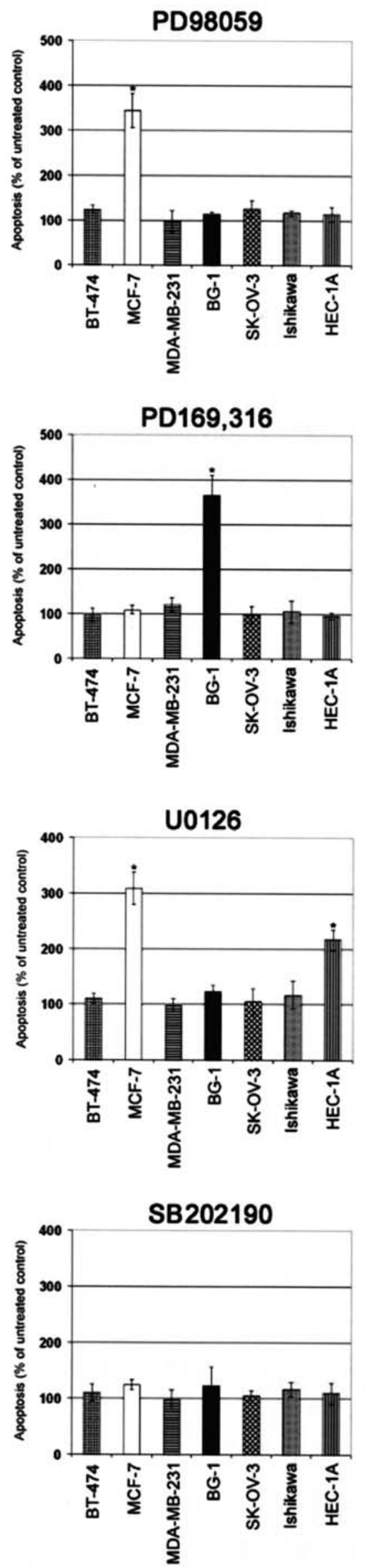

Figure 3. Apoptosis triggered by MAPK inhibitors. Apoptosis was assessed by the detection of cytoplasmic histone-DNA complexes and is expressed as a percent of the untreated control $(\mathrm{n}=4)$. * $\mathrm{p}<0.01$ vs untreated control. overactivated AKT pathway, this pathway may block apoptosis and the apoptotic action of MEK inhibitors. U0126 and PD98059 were not able to increase the apoptosis rate of BG-1 and BT-474 cells either, which exhibited the highest PTEN expression. These findings are in line with Weng et al who showed that Erk inhibitors are not able to influence cell growth if PTEN expression is high (12). In contrast to our findings, Weng et al observed a small effect of PD98059 under insulin stimulus if cellular PTEN expression was low. This discrepancy could be explained because of the insulin stimulus, which activates the Erk pathway. In this way, U0126 and PD98059, respectively, could block an overactivated pathway and thereby lead to apoptosis. The Erk inhibitors U0126 and PD98059, respectively, led to increased apoptosis of MCF-7 cells, which had a high but not maximal PTEN expression. An explanation for this effect could be the known secretion of TGF- $\alpha$ by these cells, through an autocrine loop leading to constant activation of the MAPK pathway. U0126 in contrast to PD98059 was able to increase the apoptosis rate of HEC-1A cells, which could be ascribed to the 100-fold affinity of U0126 for MEK compared to PD98059 (13).

Furthermore, we investigated the apoptosis rate in relation to PTEN expression after treatment with $\mathrm{p} 38$ kinase inhibitors PD169,316 and SB202190, respectively. The p38 kinase pathway mediates more likely proapoptotic and differentiating effects. In this manner, activation of p38 MAPK pathway leads to a release of cytochrome $\mathrm{C}$ and thereby to apoptosis. Cocolakis et al showed that PD169,136 and SB202190 can block the activin-activated p38 pathway and thereby stimulate cell growth (14). We then hypothesized that p38 kinase inhibitors might reduce the rate of apoptosis as a function of PTEN expression. However, the treatment of human cancer cells with SB202190 in comparison with untreated controls did not change the apoptosis rate significantly. Consistent with our results, Duval et al observed that by using a p38 $\alpha^{-/-}$cell line the activation of $\mathrm{p} 38 \alpha$ is not crucial in triggering apoptosis but rather a secondary event (15). It could be assumed that p38 activity was not increased in cancer cells employed in our experiments, which possibly would have allowed an obvious effect of an inhibitor. Interestingly, treatment of the BG-1 cell line, which showed high PTEN expression, with PD169,316 led to a significant increased apoptosis rate, which could not be seen in other cell lines. Sun et al reported similar results of SB203580 treated HCA-7 cells under the influence of the COX inhibitor sulindac sulfide (16). The p38 inhibitor augmented the apoptotic effect of sulindac sulfide. Thus, it is tempting to speculate that the high PTEN expression of the BG-1 cell line in combination with the p38 inhibitor PD169,316 led to an increased rate of apoptosis in our experiments. In contrast, the treatment of BG-1 cells with SB202190 did not change apoptosis significantly. Duval et al reported that SB202190 and PD169,316 have different active profiles, which could explain that discrepancy (15).

Like Erk inhibitors, p38 inhibitors are expected to have a specific activity profile, because they block 'downstream' signaling events, thereby minimizing the possibility of interactions with other signaling cascades. In contrast, tamoxifen, trastuzumab and ZD1839 block the specific receptor allowing effects on different signaling systems 
downstream the respective receptor. Clark et al showed that trastuzumab leads to Akt activation in the first $6 \mathrm{~h}$ in PTEN negative cells, which is in contrast to the expected effect (17). Only after adding a PI3-K inhibitor, apoptosis was initiated in these cells. Nagata et al reported that cells with low PTEN expression show less growth inhibition in response to treatment with trastuzumab (18). We observed similar results in our experiments. The BT-474 cell line, which had a high PTEN and Her2/neu expression, showed significantly increased apoptosis after treatment with trastuzumab compared to the untreated control. No significant change in the apoptosis rate under treatment with trastuzumab could be observed in cell lines that are Her2/neu positive, but exhibit a low or absent PTEN expression such as MCF-7, MDA-MB-231, SK-OV-3, Ishikawa and HEC-1A. Consistent with this, there was no increase in apoptosis of the PTEN high but Her2/neu low expressing BG-1 cell line. These data suggest that a combination of high PTEN and Her2/neu expression is needed for apoptotic action of trastuzumab.

Similar observations were made with regard to apoptotic action of EGFR inhibitor ZD1839. The activation of EGFR leads to phosphorylation of Akt and thereby the activation of its pathway. ZD1839 inhibits EGFR activation and as a consequence the phosphorylation of Akt. In the case of a PTEN mutation, Akt is activated independently of EGFR, which explains why ZD1839 can not influence this pathway in this specific situation. In our experiments, EGFR positive cells with high PTEN expression (BT-474, BG-1) exhibited a significant increased apoptosis after treatment with ZD1839, whereas this was not observed in cells with low PTEN expression (SK-OV-3, Ishikawa, HEC-1A). The apoptosis rate was increased in MCF-7 cells exhibiting low PTEN expression, suggesting that another pathway is involved which contributes to this effect.

It has been shown that the PI-3K/Akt pathway is able to influence ER $\alpha$ activity and thereby leads to tamoxifen resistance $(17,19)$. Our observations that tamoxifen did not increase apoptosis in $\mathrm{ER} \alpha$ positive cells with low or even no PTEN expression and therefore high activity of the Akt pathway support these findings (Fig. 2). In contrast, we were able to show a significant increase of the apoptosis rate of ER $\alpha$ and PTEN highly expressing cells. These data are in line with Shoman et al who suggested that PTEN expression is able to predict tamoxifen responsiveness (20).

In conclusion, the data of this in vitro study suggest that the apoptotic efficacy of tamoxifen, ZD1839 and trastuzumab, which act 'upstream' at receptor level, are indeed dependent on PTEN expression. This could not be shown for MAP kinase inhibitors. Further studies are required to clarify the clinical relevance of our observations.

\section{Acknowledgements}

We thank Nicole Bohnert and Constanze Siggel for their expert technical assistance.

\section{References}

1. Perry RR, Kang Y and Greaves B: Effects of tamoxifen on growth and apoptosis of estrogen-dependent and -independent human breast cancer cells. Ann Surg Oncol 2: 238-245, 1995.
2. Nahta R, Hung MC and Esteva FJ: The HER-2-targeting antibodies trastuzumab and pertuzumab synergistically inhibit the survival of breast cancer cells. Cancer Res 64: 2343-2346, 2004.

3. Mutter GL, Lin MC, Fitzgerald JT, Kum JB, Baak JP, Lees JA, Weng LP and Eng C: Altered PTEN expression as a diagnostic marker for the earliest endometrial precancers. J Natl Cancer Inst 92: 924-930, 2000.

4. Risinger JI, Hayes AK, Berchuck A and Barrett JC: PTEN/MMAC1 mutations in endometrial cancers. Cancer Res 57: 4736-4738, 1997.

5. Tsutsui S, Inoue H, Yasuda K, Suzuki K, Higashi H, Era S and Mori M: Reduced expression of PTEN protein and its prognostic implications in invasive ductal carcinoma of the breast. Oncology 68: 398-404, 2005.

6. Saito M, Okamoto A, Kohno T, Takakura S, Shinozaki H, Isonishi S, Yasuhara T, Yoshimura T, Ohtake Y, Ochiai K, Yokota $\mathrm{J}$ and Tanaka T: Allelic imbalance and mutations of the PTEN gene in ovarian cancer. Int J Cancer 85: 160-165, 2000.

7. Myers MP, Pass I, Batty IH, Van der Kaay J, Stolarov JP, Hemmings BA, Wigler MH, Downes CP and Tonks NK: The lipid phosphatase activity of PTEN is critical for its tumor supressor function. Proc Natl Acad Sci USA 95: 13513-13518, 1998.

8. Weng LP, Brown JL and Eng C: PTEN coordinates G (1) arrest by down-regulating cyclin D1 via its protein phosphatase activity and up-regulating p27 via its lipid phosphatase activity in a breast cancer model. Hum Mol Genet 10: 599-604, 2001.

9. Gu J, Tamura M, Pankov R, Danen EH, Takino T, Matsumoto K and Yamada KM: Shc and FAK differentially regulate cell motility and directionality modulated by PTEN. J Cell Biol 146: 389-403, 1999.

10. Gu J, Tamura M and Yamada KM: Tumor suppressor PTEN inhibits integrin- and growth factor-mediated mitogen-activated protein (MAP) kinase signaling pathways. J Cell Biol 143: 1375-1383, 1998.

11. DeGraffenried LA, Fulcher L, Friedrichs WE, Grunwald V, Ray RB and Hidalgo M: Reduced PTEN expression in breast cancer cells confers susceptibility to inhibitors of the PI3 kinase/Akt pathway. Ann Oncol 15: 1510-1516, 2004.

12. Weng LP, Smith WM, Brown JL and Eng C: PTEN inhibits insulin-stimulated MEK/MAPK activation and cell growth by blocking IRS-1 phosphorylation and IRS-1/Grb-2/Sos complex formation in a breast cancer model. Hum Mol Genet 10: 605-616, 2001.

13. Favata MF, Horiuchi KY, Manos EJ, Daulerio AJ, Stradley DA, Feeser WS, Van Dyk DE, Pitts WJ, Earl RA, Hobbs F, Copeland RA, Magolda RL, Scherle PA and Trzaskos JM: Identification of a novel inhibitor of mitogen-activated protein kinase kinase. J Biol Chem 273: 18623-18632, 1998.

14. Cocolakis E, Lemay S, Ali S and Lebrun JJ: The p38 MAPK pathway is required for cell growth inhibition of human breast cancer cells in response to activin. J Biol Chem 276: 18430-18436, 2001.

15. Duval D, Malaise M, Reinhardt B, Kedinger C and Boeuf H: A p38 inhibitor allows to dissociate differentiation and apoptotic processes triggered upon LIF withdrawal in mouse embryonic stem cells. Cell Death Differ 11: 331-341, 2004.

16. Sun Y and Sinicrope FA: Selective inhibitors of MEK1/ ERK44/42 and p38 mitogen-activated protein kinases potentiate apoptosis induction by sulindac sulfide in human colon carcinoma cells. Mol Cancer Ther 4: 51-59, 2005.

17. Clark AS, West K, Streicher S and Dennis PA: Constitutive and inducible Akt activity promotes resistance to chemotherapy, trastuzumab, or tamoxifen in breast cancer cells. Mol Cancer Ther 1: 707-717, 2002.

18. Nagata Y, Lan KH, Zhou X, Tan M, Esteva FJ, Sahin AA, Klos KS, Li P, Monia BP, Nguyen NT, Hortobagyi GN, Hung MC and Yu D: PTEN activation contributes to tumor inhibition by trastuzumab and loss of PTEN predicts trastuzumab resistance in patients. Cancer Cell 6: 117-127, 2004.

19. Faridi J, Wang L, Endemann G and Roth RA: Expression of constitutively active Akt-3 in MCF-7 breast cancer cells reverses the estrogen and tamoxifen responsivity of these cells in vivo. Clin Cancer Res 9: 2933-2939, 2003.

20. Shoman N, Klassen S, McFadden A, Bickis MG, Torlakovic E and Chibbar R: Reduced PTEN expression predicts relapse in patients with breast carcinoma treated by tamoxifen. Mod Pathol 18: 250-259, 2005. 